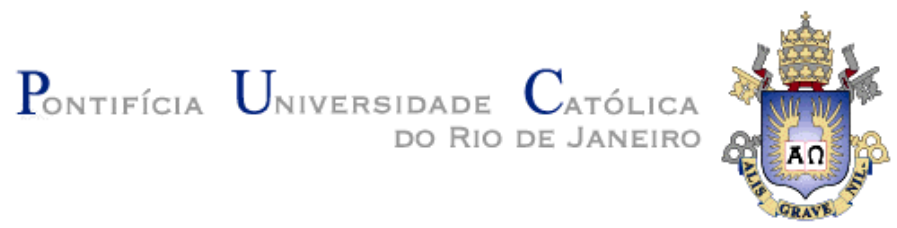

Tanara Lauschner

Modelagem de restrições de esquemas mediados

Tese de Doutorado

Tese apresentada como requisito parcial para obtenção do título de Doutor pelo Programa de PósGraduação em Informática da PUC-Rio.

Orientador: Prof. Marco Antonio Casanova 


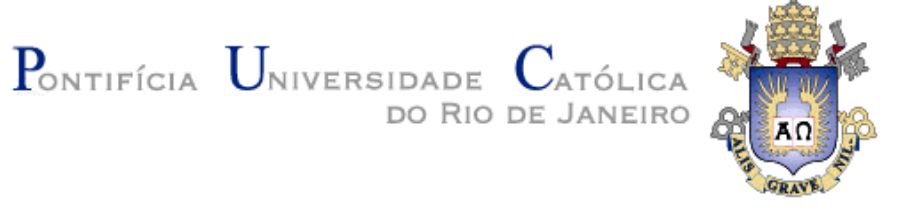

Tanara Lauschner

\section{Modelagem de restrições de esquemas mediados}

Tese apresentada como requisito parcial para obtenção do título de Doutor pelo Programa de Pós-Graduação em Informática da PUC-Rio. Aprovada pela Comissão Examinadora abaixo assinada.

Prof. Marco Antonio Casanova Departamento de Informática - PUC-Rio (Orientador)

Prof. Antonio Luz Furtado Departamento de Informática - PUC-Rio (Co-orientador)

Profa. Karin Koogan Breitman Departamento de Informática - PUC-Rio

Prof. Altigran Soares da Silva Departamento de Ciência da Computação - UFAM

Prof ${ }^{a}$. Vânia Maria Ponte Vidal Departamento de Computação - UFC

Prof. José Antônio F. de Macedo Departamento de Computação - UFC

Luiz André P. Paes Leme Instituto de Computação - UFF 
Todos os direitos reservados. É proibida a reprodução total ou parcial do trabalho sem autorização da universidade, da autora e do orientador.

Tanara Lauschner

Mestre em Ciência da Computação pela UFMG(Universidade Federal de Minas Gerais) em fevereiro de 2002. Graduou-se em Engenharia Elétrica pela UFAM (Universidade Federal do Amazonas) em março de 1998.

Ficha Catalográfica

Lauschner, Tanara

Modelagem de restrições de esquemas mediados/ Tanara Lauschner; orientador: Marco Antonio Casanova. Rio de Janeiro: PUC-Rio, Departamento de Informática, 2010.

v., 80 f.: il. ; $29,7 \mathrm{~cm}$

1. Tese (Doutorado em Informática) - Pontifícia Universidade Católica do Rio de Janeiro, Departamento de Informática.

Inclui bibliografia

1. Informática - Teses 2. Restrições de esquemas mediados. 3. Esquema mediado. 4. Lógica de Descrição. 5. Bancos de Dados. I. Casanova, Marco Antonio. II. Pontifícia Universidade Católica do Rio de Janeiro. Departamento de Informática. III. 


\section{Resumo}

Lauschner, Tanara; Casanova, Marco Antonio. Modelagem de restrições de esquemas mediados. Rio de Janeiro, 2010. 80p. Tese de Doutorado Departamento de Informática, Pontifícia Universidade Católica do Rio de Janeiro.

Integração de dados refere-se ao problema de combinar dados que estão armazenados em diferentes fontes, fornecendo ao usuário uma visão unificada dos dados. As consultas são então expressas em um esquema global ou esquema mediado, que deve incluir restrições de integridade que contribuam para um entendimento correto sobre o que a semântica das fontes de dados do ambiente de mediação tem em comum. Esta tese endereça o problema de modelar as restrições de um esquema mediado a partir das restrições dos esquemas importados e dos mapeamentos de esquemas. Argumenta-se que as restrições devem ser modeladas como o ínfimo das restrições dos esquemas exportados, depois de traduzidos para um vocabulário comum. Desta forma, assegura-se que os usuários do esquema mediado interpretarão os resultados das consultas corretamente. Para uma família expressiva de restrições, esta tese mostra como computar eficientemente o ínfimo de conjuntos de restrições.

\section{Palavras-chave}

Restrições de esquemas mediados, esquemas mediados, lógica de descrição. 


\section{Abstract}

Lauschner, Tanara; Casanova, Marco Antonio. Modeling the Mediated Schema Constraints. Rio de Janeiro, 2010. 80p. DSc. Thesis Departamento de Informática, Pontifícia Universidade Católica do Rio de Janeiro.

Data integration refers to the problem of combining data stored in different sources, providing users with a unified view of the data. Queries are then expressed in terms of a global or mediated schema, which should include integrity constraints that contribute to a correct understanding of what the semantics of the data sources have in common. This thesis addresses the problem of modeling the constraints of a mediated schema from the imported schemas constraints and mappings. It argues that the constraints should be modeled as the greatest lower bound of the constraints of the export schemas, after appropriate translation to a common vocabulary. This assures that users of the mediated schema will correctly interpret query results. For a rich family of constraints, it shows how to efficiently compute the greatest lower bound of sets of constraints.

\section{Keywords}

Integrity constraint, mediated schema, description logics. 


\section{Sumário}

1 Introdução 11

1.1. Integração de dados e ambiente de mediação 11

1.2. O problema de revisar as restrições de um esquema mediado 13

1.3. Contribuições 15

1.4. Trabalhos relacionados 16

$\begin{array}{ll}\text { 1.5. Organização da tese } & 18\end{array}$

2 Esquemas conceituais em lógica de descrição 20

2.1. Uma família de linguagens atributivas 20

2.2. Esquemas elementares 23

2.3. Esquemas ultra elementares $\quad 27$

2.4. Ambiente de mediação $\quad 28$

2.5. Conclusões do capítulo 34

3 Problema de decisão em esquemas conceituais $\quad 35$

3.1. Inclusão de consultas e problemas de decisão correlatos 36

3.2. Extensão do tableau para esquemas ultra elementares 39

3.3. Um procedimento de decisão rápido 43

3.4. Conclusões do capítulo 48

4 Construção das restrições do esquema mediado 50

4.1. Passos básicos do processo de revisão das restrições 50

4.2. Cálculo do ínfimo de dois conjuntos de restrições 62

4.3. Conclusões do capítulo 72

5 Conclusões e trabalhos futuros $\quad 74$

$\begin{array}{ll}6 \text { Referências bibliográficas } & 76\end{array}$ 


\section{Lista de figuras}

Figura 1 - Componentes de um ambiente de mediação .................................. 12

Figura 2 - Definição informal do esquema da Amazon................................... 26

Figura 3 - Definição informal do esquema do eBay....................................... 26

Figura 4 - Definição formal das restrições do esquema da Amazon .................. 27

Figura 5 - Definição formal de algumas das restrições do esquema do eBay ...27

Figura 6 - Vocabulário do esquema mediado Sales ........................................ 30

Figura 7 - Vocabulário do esquema importado da Amazon ...............................30

Figura 8 - Restrições do esquema importado da Amazon ................................. 31

Figura 9 - Mapeamento local do esquema da Amazon ................................... 31

Figura 10 - Vocabulário do esquema importado eBay ................................... 32

Figura 11 - Restrições do esquema importado eBay .................................... 32

Figura 12 - Mapeamento local do esquema eBay ........................................ 32

Figura 13 - Restrições do esquema mediado Sales .................................... 32

Figura 14 - Mapeamento de mediação do esquema Sales ............................... 32

Figura 15 - Ambiente de mediação Sales com o esquema da Amazon............. 33

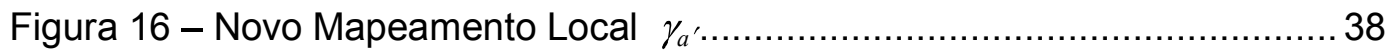

Figura 17 - Regras adicionais do tableau ................................................... 40

Figura 18 - Exemplo de um tableau .......................................................... 42

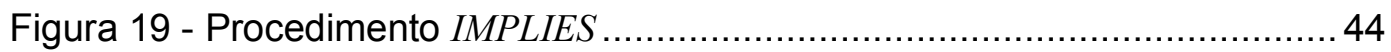

Figura 20 - Grafo de dependência estendido ............................................ 46

Figura 21 - Criação do Novo Esquema Mediado.......................................... 51

Figura 22 - Mapeamento de mediação revisado ............................................5 56

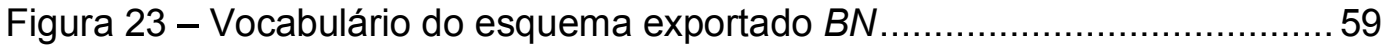

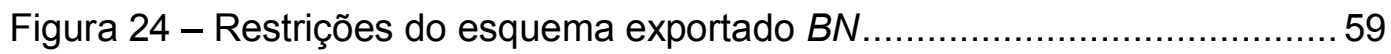

Figura 25 - Vocabulário do esquema mediado Sales/BN ...............................59

Figura 26 - Vocabulário do esquema mediado Sales ................................... 59

Figura 27 - Vocabulário do Esquema Importado $B N$........................................6 60

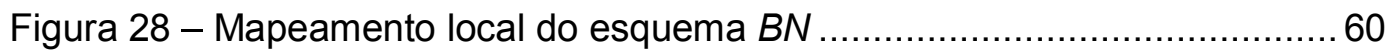

Figura 29 - Mapeamento de mediação do ambiente de mediação Sales/BN ....61 61

Figura 30 - Restrições do esquema importado de $B N$.................................... 61

Figura 31 - Restrições do esquema mediado revisado Sales/BN.....................62

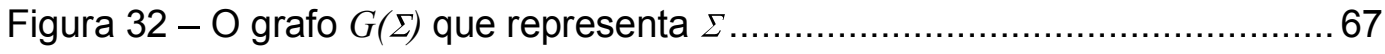

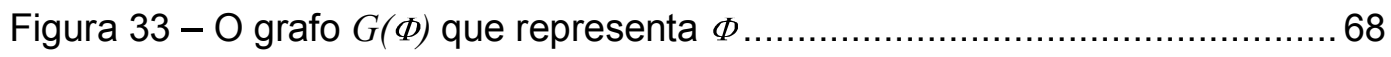

Figura 34 - Construção de $\Gamma$ que gera o ínfimo de $\Sigma$ e $\Phi \ldots \ldots \ldots \ldots \ldots \ldots \ldots \ldots \ldots \ldots . \ldots . \ldots . \ldots \ldots$ 


\section{Lista de exemplos}

Exemplo 2-1 - Esquemas Amazon e eBay .................................................. 26

Exemplo 2-2 - Ambiente Mediado Sales ...................................................... 30

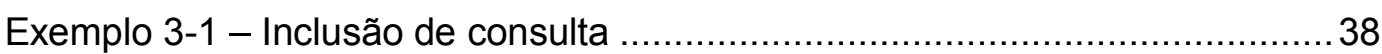

Exemplo 3-2 - Consulta Eficaz .................................................................. 38

Exemplo 3-3 - Restrições de subconjunto do esquema importado ..................... 39

Exemplo 3-4 - Resolução das restrições de subconjunto com tableau ...............42 42

Exemplo 3-5 - Aplicação do procedimento IMPLIES ao Exemplo 3-4 ................ 46

Exemplo 3-6 - Problema de inclusão de consulta com IMPLIES ........................46

Exemplo 4-1 - Definição das restrições do esquema mediado. ..........................55

Exemplo 4-2 - Restrições que geram resultado vazio. ....................................63

Exemplo 4-3 - Geração do grafo $G(\Sigma, \Omega)$ que representa $\Sigma$...........................66

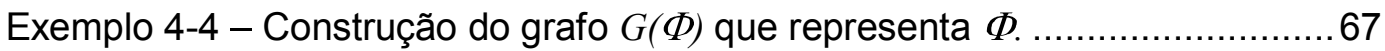

Exemplo 4-5 - Formalização do Passo C do. Exemplo 4-1 ...............................70 


\section{Lista de símbolos}

$\mathcal{L} \quad$ Linguagem atributiva

$\mathcal{A} \quad$ Alfabeto da linguagem atributiva

$\mathrm{T} \quad$ Conceito/papel universal ou supremo (universal concept/role)

$\perp \quad$ Conceito/papel vazio ou ínfimo (bottom concept/role)

$(p \circ q) \quad$ Composição

$p^{-} \quad$ Inversa

$\neg e \quad$ Negação

$e \sqcap f \quad$ Interseção

$e \sqcup f \quad$ União

$\exists p \quad$ Quantificação existencial restrita (restricted existential quantification)

$\exists$ p.e Quantificação existencial irrestrita (full existential quantification)

$\forall p . e \quad$ Restrição de valor ou quantificação universal irrestrita

$(\leq n p) \quad$ Restrição de máximo (at-most restriction)

( $\geq n p) \quad$ Restrição de mínimo (at-least restriction)

$\boldsymbol{S}$ Interpretação para os símbolos do alfabeto $\mathcal{A}$

$\Delta^{s} \quad$ Domínio da interpretação $s$

$u \sqsubseteq v \quad$ Inclusão

$u \mid v \quad$ Disjunção

$u \equiv v \quad$ Equivalência

$\mathbf{s} \models \sigma \quad$ Interpretação $s$ satisfaz a fórmula $\sigma$

$\mathbf{s} \models \Sigma \quad$ Interpretação $s$ satisfaz todas as formulas do conjunto de fórmulas $\Sigma$

$\Sigma \models \sigma \quad$ Conjunto de fórmulas $\Sigma$ implica logicamente uma fórmula $\sigma$

$\operatorname{Th}(\Sigma) \quad$ Teoria induzida por $\Sigma$

$M \quad$ Ambiente de mediação

MV Vocabulário de $M$

MC Conjunto de restrições de $M$

$E_{i} \quad$ Esquema exportado da fonte de dados $i$

$E V_{i} \quad$ Vocabulário de $E_{i}$

$E C_{i} \quad$ Conjunto de restrições de $E_{i}$ 
$\begin{array}{ll}I_{i} & \text { Esquema importado referente ao } E_{i} \\ I V_{i} & \text { Vocabulário de } I_{i} \\ I C_{i} & \text { Conjunto de restrições de } I_{i} \\ \gamma & \text { Mapeamento de mediação (mediated mapping) } \\ \bar{\gamma} & \text { Função induzida por } \gamma \\ \gamma_{k} & \text { Mapeamento local (local mapping) } \\ \bar{\gamma}_{k} & \text { Função induzida por } \gamma_{k} \\ \Phi_{1} \triangle \Phi_{2} & \text { Ínfimo ou maior limite inferior (greatest lower bound - g.l.b.) } \\ \Phi_{1} \nabla \Phi_{2} & \text { Supremo ou menor limite superior (least upper bound - l.u.b.) }\end{array}$ 